\title{
Characterization of the Auxin Efflux Transporter PIN Proteins in Pear
}

\author{
Liying Qi ${ }^{1,2,+}$, Ling Chen ${ }^{1,+} \mathbb{C}$, Chuansen Wang ${ }^{1}$, Shaoling Zhang ${ }^{3}$, Yingjie Yang ${ }^{1}$, \\ Jianlong Liu ${ }^{1}$, Dingli Li ${ }^{1}$, Jiankun Song ${ }^{1}$ and Ran Wang ${ }^{1, *}$ \\ 1 College of Horticulture, Qingdao Key Lab of Genetic Improvement and Breeding of Horticultural Plants, \\ Qingdao Agricultural University, Qingdao 266109, China; qiliying88@163.com (L.Q.); \\ 18254220858@163.com (L.C.); m17864291519@163.com (C.W.); wuhuaguoyyj@163.com (Y.Y.); \\ liujianlong1357@163.com (J.L.); qauldl@163.com (D.L.); qausjk@126.com (J.S.) \\ 2 Institute of Soil and Fertilizer \& Resource and Environment, Jiangxi Academy of Agriculture Sciences, \\ Nanchang 330200, China \\ 3 College of Horticulture, Nanjing Agricultural University, Nanjing 210095, China; slzhang@njau.edu.cn \\ * Correspondence: qauwr@126.com \\ + Those authors contributed equally to this work.
}

Received: 16 January 2020; Accepted: 4 March 2020; Published: 10 March 2020

check for updates

\begin{abstract}
PIN-FORMED (PIN) encodes a key auxin polar transport family that plays a crucial role in the outward transport of auxin and several growth and development processes, including dwarfing trees. We identified a dwarfing pear rootstock 'OHF51' (Pyrus communis), which limits the growth vigor of the 'Xueqing' (Pyrus bretschneideri $\times$ Pyrus pyrifolia) scion, and isolated 14 putative PbPINs from the pear Pyrus bretschneideri. The phylogenic relationships, structure, promoter regions, and expression patterns were analyzed. PbPINs were classified into two main groups based on the protein domain structure and categorized into three major groups using the neighbor-joining algorithm. Promoter analysis demonstrated that PbPINs might be closely related to plant growth and development. Through quantitative real-time PCR (qRT-PCR) analysis, we found that the expression patterns of 14 PbPINs varied upon exposure to different organs in dwarfing and vigorous stocks, 'OHF51' and 'QN101' (Pyrus betulifolia), indicating that they might play varying roles in different tissues and participated in the regulation of growth vigor. These results provide fundamental insights into the characteristics and evolution of the PINs family, as well as the possible relationship between dwarfing ability and auxin polar transport.
\end{abstract}

Keywords: Pyrus; auxin transport; dwarfing

\section{Introduction}

Dwarfing and high plant population density are the inevitable trends of fruit production owing to their important features such as earlier fruiting, higher yields, and ease of management. Currently, application of growth-regulating agents is sometimes used to control tree vegetative growth [1-4] and many dwarfing rootstocks or interstocks of fruit trees are also widely used to control tree size [5-8]. Despite its broad utility and the availability of related research on dwarfing rootstocks/interstocks, the underlying mechanism of rootstock/interstock-induced dwarfing is still unknown.

It has been suggested that the dwarfing physiological mechanism might be related to the vessel dimensions, hormone metabolism, and phenol contents of rootstocks [9], as well as the disorder of vascular organization reestablishment and the resultant decline in material transport capacity in the graft union.

In apple trees, it has been reported that the content, transport, and signaling of hormones might play roles in rootstock/interstock-induced dwarfing. For example, the Gibberellic Acid (GA) content 
in the xylem of dwarfing rootstock ' $\mathrm{M} 9$ ' is lower than that of vigorous rootstocks [10,11], and the application of GA to scions on 'M9' increased node number [12]. Abscisic Acid (ABA) content is also higher in dwarfing rootstocks $[13,14]$. Indole Acetic Acid (IAA)content in leaves and branch-barks of scions grafted on the dwarfing interstocks were lower than that of invigorating combinations, but after bridging and substituting interstock bark with invigorating interstock, IAA content in scion gradually restored to a standard tree's level $[15,16]$. When auxin transport in the stem was inhibited by polar auxin inhibitor 1-N-naphthylphtha-lamic acid (NPA) in vigorous rootstocks, root zeatin level, and shoot growth declined dramatically [17]. Thus, at present, the best-supported model proposes that dwarfing rootstocks decrease auxin polar transport, thereby reducing root growth and the amount of root-synthesized cytokinin supplied to the scion, which in turn limit the growth of the canopy $[9,13]$. The auxin polar transport is mainly mediated through auxin carriers, including AUXIN RESISTENT1/LIKE AUX1 (AUX/LAX) influx carrier, PIN-FORMED (PIN) efflux carriers, and ATP binding, cassette B/P-glycoprotein/Multidrug-resistance (ABCB/MDR/PGP) efflux/condition carriers [18-20].

The different contents of IAA have been shown to possibly result from the expression levels of the PIN gene family members [21]. The PIN-FORMED (PIN) auxin efflux carriers are the major proteins that control polar auxin transport (PAT), which is the unidirectional transporting IAA from the top of the plant morphology to the lower parts [22-25]. PIN proteins have generally been investigated in Arabidopsis thaliana. To date, eight PIN members (AtPIN1 to AtPIN8) have been reported [26] and divided into two categories according to the cell localization. AtPIN1, 2, 3, 4, and 7 are localized on the plasma membrane (PM), mainly responsible for regulating intercellular auxin movement of plants. On the other hand, AtPIN5 and AtPIN8 are localized in the endoplasmic reticulum (ER) $[27,28]$ and have been reported to balance cellular auxin homeostasis. At the same time, PIN proteins have a conserved structure, with trans-membrane helices at the $\mathrm{N}$ and $\mathrm{C}$ terminals, and some contain an intracellular hydrophilic loop domain [29]. Therefore, based on the presence of the central hydrophilic loop domain, the eight AtPINs can be divided into another two categories. AtPIN1, 2, 3, 4, and 7, with a long hydrophilic loop separating two extremely conserved hydrophobic domains are categorized as canonical PINs. AtPIN5 and AtPIN8 with the absence of the central hydrophilic loop belong to the non-canonical PINs category. AtPIN6 has been reported to be different from other PINs as it is localized on the plasma membrane as well as endoplasmic reticulum [30]. In addition, the hydrophobic loop of PIN6 appeared to have Ser/Thr phosphorylation sites, which were usually a feature of canonical PINs.

PIN proteins have been indicated to play various roles in plant developmental processes [19,31], including embryo development [32,33], lateral root development, shoot vascular development, and floral bud formation, as well as hormone and abiotic stress responses [34-36]. For example, AtPIN1 is mainly involved in the maintenance of embryonic auxin gradients, and organ initiation is severely affected in pin1 mutants, which results in the formation of naked inflorescence stems [16]. PIN2 has been reported to be the pivotal PIN in regulating root meristem swelling [16]. In rice, the OsPIN1b gene has been reported to play roles in auxin-dependent adventitious root emergence and tillering [34]. The transport of auxin from shoot to the root-shoot junction reportedly increased together with tiller number in OsPIN2 overexpressing plants and resulted in lowered plant height [37]. OsPIN3t, is involved in drought stress response and drought tolerance [38]. In addition, OsPIN9, OsPIN10a, and OsPIN10b, are expressed at high levels in adventitious root primordia and pericyclic cells at the stem base, suggesting that they might be involved in adventitious root development. In maize, the expression levels of most ZmPIN genes were induced in shoots and reduced in roots by various abiotic stress treatments [34,35]. ZmPIN1 $a$ and ZmPIN1b analysis has also established their involvement in endosperm and embryonic development $[39,40]$. Eleven PIN genes have been identified in sorghum and all SbPIN genes were induced by IAA [41].

To date, a series of PIN family members have been reported in some species, including Solanum lycopersicum [42], Glycine max [43], Nicotiana tabacum [44], Brassica rapa [45], and Citrullus lanatus [15], as well as some perennial woody plants, such as Vitis vinifera [25], Populus trichocarpa [46], and 
Eucalyptus [47]. Their functions are similar, with some related to root development and most responding to auxins or abiotic stress. However, there is almost no research on PIN genes related to dwarfing; there are no genome-wide analyses of PIN gene families in Pyrus. In this study, we performed a genome-wide analysis of the PIN family in pear and analyzed the expression profiles of PbPINs in different organs of dwarfing and vigorous rootstocks: 'QN101' (Pyrus betulifolia) and 'OHF51' (Pyrus communis). As the scion used in those pear-grafting combinations was 'Xueqing,' which came from crosses between 'Xuehuali' (Pyrus bretschneideri) and 'Nijisseiki' (Pyrus pyrifolia), the Pyrus bretschneideri genome was selected for PIN-FORMED (PIN) identification. To our knowledge, this is the first study focusing on the identification and expression patterns of PbPINs. Our data provides a solid basis for future functional genomic studies on the PIN gene family and further research on the dwarfing mechanism. In addition, the identification of PIN genes in Pyrus will help in understanding the molecular structure and evolution history of PIN genes in plants.

\section{Results}

\subsection{Dwarfing Pear Rootstock 'OHF51' Limits the Growth Vigor of the Scion}

Fruit quality and tree structural data were collected from two different pear-grafting combinations, 'Xueqing'/'QN101'/'Douli' and 'Xueqing'/'OHF51'/'Douli.' As shown in Table 1, when the mean fruit weight was $537.0 \mathrm{~g}$ in the combination of 'OHF51' used as interstock, which was greater than that in the combination of 'QN101' used as interstock (443.2 g). The total soluble solids (TSS) and firmness of fruits between two combinations showed no markedly difference. The tree height was lesser in 'OHF51' combination (2.62 $\mathrm{m}$ ) than that in 'QN101' combination (2.93 m) (Table 1). The trunk diameter of scion grafted on 'OHF51' was thinner than that grafted on 'QN101.' Moreover, the diameter ratios of scion/interstock/rootstock were also different between the two grafting combinations, which were 0.76/1/0.96 in the 'OHF51' combination and 0.96/1/1.14 in the 'QN101' combination. Those results indicated that 'OHF51,' which is a pear dwarfing stock, could limit the growth vigor of the scion and affect the growth of rootstock. However, scion grafted on 'OHF51' could lead to increased fruit yield.

Table 1. Comparison of two types of grafting combinations.

\begin{tabular}{lcc}
\hline \multicolumn{1}{c}{ Grafting Combinations } & 'Xueqing'/'QN101'/'Douli' & 'Xueqing'/'OHF51'/'Douli' \\
\hline Fruit weight (g) & $443.2+16.0 \mathrm{~b}$ & $537.0 \pm 29.6 \mathrm{a}$ \\
Total Soluble Solids (TSS) content & $13.73 \pm 0.16 \mathrm{a}$ & $13.56 \pm 0.16 \mathrm{a}$ \\
Firmness $\left(\mathrm{Kg} \mathrm{cm}{ }^{-2}\right.$ ) & $6.53 \pm 0.12 \mathrm{a}$ & $6.42 \pm 0.16 \mathrm{a}$ \\
Tree height $(\mathrm{m})$ & $2.93 \pm 0.08 \mathrm{a}$ & $2.62 \pm 0.08 \mathrm{~b}$ \\
Scion diameter (mm) & $42.77 \pm 2.62 \mathrm{a}$ & $33.54 \pm 0.98 \mathrm{~b}$ \\
Interstock diameter (mm) & $44.63 \pm 2.12 \mathrm{a}$ & $44.10 \pm 1.41 \mathrm{a}$ \\
Rootstock diameter (mm) & $50.78 \pm 2.88 \mathrm{a}$ & $43.46 \pm 2.50 \mathrm{a}$ \\
Diameter ratio of trunk & $0.96 / 1 / 1.14$ & $0.76 / 1 / 0.96$ \\
amongScion/Interstock/Rootstock & & \\
\hline
\end{tabular}

Note: Data are presented by mean \pm SEM (Standard Error of Mean). Means followed by different letters are significantly different at the $P<0.05$ level using the Students' $t$-test.

\subsection{Genome-Wide Identification of PIN Genes in Pyrus Bretschneideri}

In order to understand if the dwarfing ability of rootstocks was related to the decrease of auxin polar transport, 14 putative PbPINs in Pyrus were identified. Upon searching SMART (Simple Modular Architecture Research Tool) and Pfam databases using the corresponding protein sequences, the 14 PbPINs were all confirmed to encode memtrans-containing proteins, which belong to the PIN family. Related information on gene locus IDs, genomic positions, coding region lengths, and the corresponding proteins of PbPIN genes are listed in Table 2. 
Table 2. Characteristics of PIN members in pear

\begin{tabular}{|c|c|c|c|c|c|c|c|c|}
\hline Gene Name & Gene Locus ID & Genomic Position & CDS Length & Protein Length & $\begin{array}{c}\text { Molecular } \\
\text { Weight (Da) }\end{array}$ & $\begin{array}{c}\text { Isoelectric } \\
\text { Point (PI) }\end{array}$ & $\begin{array}{l}\text { Instability } \\
\text { Index }\end{array}$ & Aliphatic Index \\
\hline PbPIN1-1 & LOC103946937 & $824,136 . .828,039$ & 1863 & 620 & $67,663.97$ & 9.11 & 34.52 & 88.55 \\
\hline PbPIN1-2 & LOC103950573 & $343,331 . .347,039$ & 1869 & 622 & $67,713.01$ & 8.98 & 34.59 & 88.62 \\
\hline PbPIN1-3 & LOC103933990 & $216,016 . .218,808$ & 1854 & 617 & $66,514.59$ & 8.89 & 37.38 & 94.05 \\
\hline PbPIN1-4 & LOC103960490 & $472,382 . .474,775$ & 1761 & 586 & $63,204.43$ & 9.11 & 40.01 & 88.72 \\
\hline PbPIN2-1 & LOC103941631 & 34496.38649 & 1944 & 647 & $69,852.83$ & 9.32 & 42.75 & 89.68 \\
\hline PbPIN2-2 & LOC103950477 & $728,493.732,110$ & 1944 & 647 & $69,832.76$ & 9.25 & 43.06 & 89.98 \\
\hline PbPIN3-1 & LOC103947028 & $312,759 . .317,361$ & 1980 & 659 & $71,419.31$ & 8.47 & 36.62 & 90.17 \\
\hline PbPIN3-2 & LOC103948593 & $3,901,582 . .3,905,923$ & 1917 & 638 & $69,255.79$ & 8.2 & 33.77 & 92.66 \\
\hline PbPIN3-3 & LOC103948670 & $4,098,128 . .4,102,477$ & 1917 & 638 & $69,255.79$ & 8.2 & 33.77 & 92.66 \\
\hline PbPIN4 & LOC103931858 & $51256 . .56492$ & 1857 & 618 & $68,224.38$ & 9.54 & 38.8 & 93.12 \\
\hline PbPIN5-1 & LOC103930394 & $305,314 . .313,840$ & 1080 & 359 & $39,296.44$ & 7.65 & 35.98 & 116.71 \\
\hline PbPIN5-2 & LOC103938552 & $149,838 . .151,159$ & 1080 & 359 & $39,489.7$ & 7.61 & 35.38 & 115.35 \\
\hline PbPIN6 & LOC103951142 & $485,530 . .492,630$ & 1662 & 553 & $60,371.48$ & 8.93 & 39.93 & 98.43 \\
\hline PbPIN8 & LOC103934837 & $52,931 . .55,068$ & 1074 & 357 & $38,888.24$ & 9.29 & 30.69 & 120.25 \\
\hline
\end{tabular}




\subsection{Phylogenetic Relationship Analysis of the PbPIN Family Genes}

Several studies have investigated the biological functions of the PIN auxin efflux carriers in Arabidopsis [48,49]. Investigation of the evolutionary relationships of PINs from P. bretschneideri and Arabidopsis will aid in understanding the possible biological functions of these auxin carriers in Pyrus.

Phylogenetic analysis of 22 PIN proteins, including eight AtPINs and 14 PbPINs, was carried out using MEAG7.0 software using the neighbor-joining method. The results showed that most PbPINs were clustered with their orthologs in Arabidopsis with a high bootstrap support, such as PIN2s, PIN5s, PIN6s, and PIN8s (Figure 1). Some paralogs also existed in pear-like PbPIN1-4/PbPIN1-3, PbPIN2-1/PbPIN2-2, and PbPIN5-1/PbPIN5-2. Adopting the homology of the family members of Arabidopsis, the PIN family members of pear were systematically named PbPIN1-1 to PbPIN8, respectively.

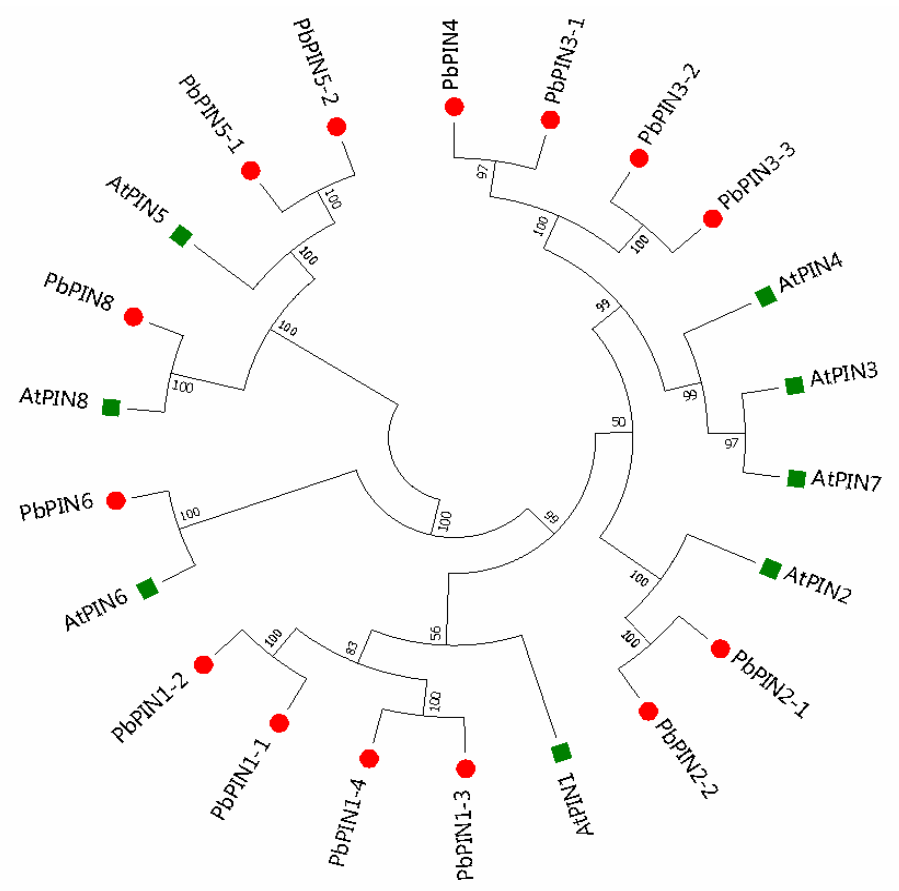

Figure 1. Phylogenetic relationships of PINs between Pyrus bretschneideri and Arabidopsis. Complete alignments of 22 PINs from P. bretschneideri and Arabidopsis were performed to construct a phylogenetic tree using MEGA 7.0 software with the neighbor-joining method. Bootstrap values are indicated on the nodes of branches. The green dots and red squares represent AtPINs and PbPINs, respectively. The 14 $\mathrm{PbPINs}$ were denominated according to the sequence alignments and the phylogenetic tree.

\subsection{Exon-Intron Structure of PbPINs}

To investigate the structures of the PIN genes, the numbers and locations of exons and introns were identified using GSDS v2.0 (http://gsds.cbi.pku.edu.cn/) (Figure 2).

Although the number of exons among these PbPIN genes is different, changing from three to eight, the genetic structures of most PbPINs are highly conserved, and seven out of the 14 PbPINs all contained six exons. In addition, these PbPINs were categorized into four major groups using the neighbor-joining algorithm. The six genes clustered into Group I exhibited similar exon-intron structures: five members contained six exons, except for PbPIN4, which contained seven exons. However, the members of Groups II and IV contained different numbers of exons. In Groups II, PbPIN1-3 and PbPIN1-4 had five exons, and PbPIN1-1 and PbPIN1-2 had six exons. In Groups IV, PbPIN5-1 and PbPIN5-2 both had three exons, and PbPIN8 contained seven exons. PbPIN6, the only one member in group III, had eight exons. The number of introns implied different lengths of these genes. In addition, the sequence lengths of intron or untranslated region were variable, which also affected the length of genes. For 
example, PbPIN5-1, which was approximately $9 \mathrm{~Kb}$ long, had a long untranslated region, and PbPIN6 harbored a relatively longer intron.

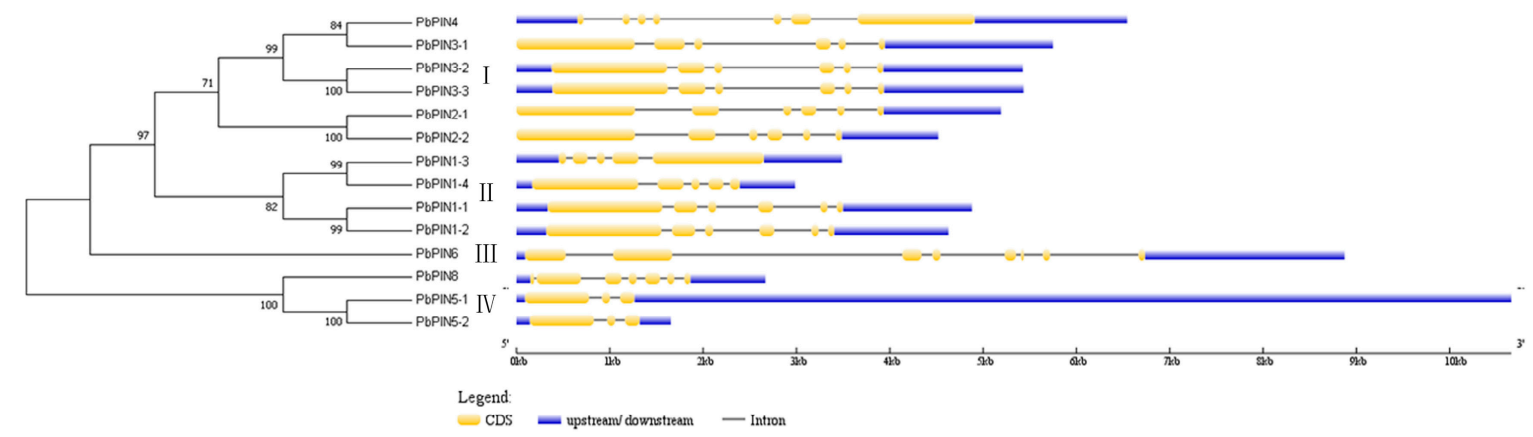

Figure 2. Phylogenetic relationship and exon-intron structure of PbPINs in Pyrus. The neighbor-joining tree was constructed using MEGA 7.0. Bootstrap values are indicated on the nodes of branches. The yellow boxes denote CDS (Coding Sequence), the blue lines show UTR (Untranslated Regions), and the black lines represent intron.

\subsection{Analysis of Conservative Domain of PIN Family}

The length of PIN family members varied widely. We categorized all members into two groups: the long PbPINs (PbPIN1s, PbPIN2s, PbPIN3s, PbPIN4, and PbPIN6), and the short PbPINs (PbPIN5-1, PbPIN5-2, and PbPIN8) (Figure 3).

The different members contained one or two highly conservative transmembrane domains (TMDs), which comprised 200, 350, or 500 amino acids. Meanwhile, similar to other plant PINs, PbPIN proteins have a highly conserved hydrophobicity profile with 7-9 transmembrane helices located at $\mathrm{N}$ and C-termini, and linked by a central hydrophilic loop (Figure 4). The length of the central hydrophilic loop is around 300 amino acids for long members of PbPINs and 50 amino acids for short members of PINs (PIN5-1, PIN5-2, and PIN8).

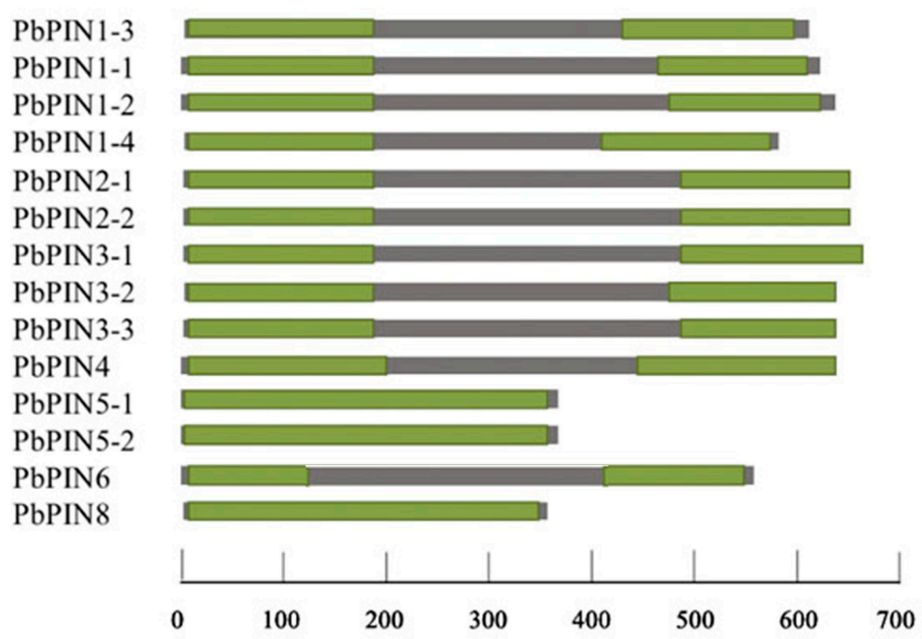

Figure 3. Structures of PbPINs in pear. The name of each corresponding protein is shown on the left. The green color indicates the position of the corresponding conserved domains: transmembrane domains (TMDs). The scale bar represents 100 amino acids. 

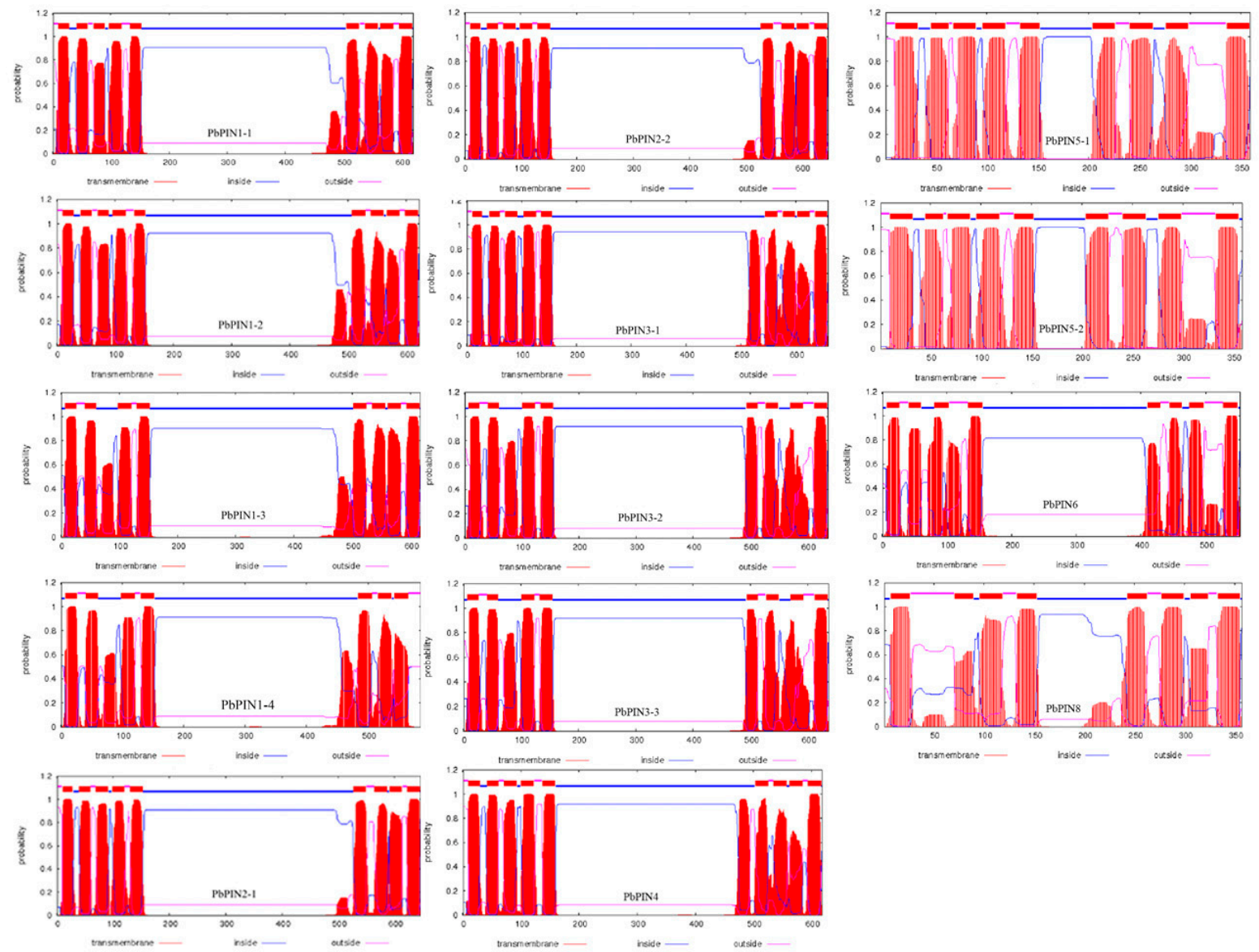

Figure 4. Transmembrane topology analysis of pear PbPIN proteins. The protein transmembrane topology was predicted by using the TMHHM Server v2.0. The predicted transmembrane helices are shown as red peaks.

\subsection{Promoter Analysis of PbPINs}

Specific cis-regulatory elements present in the promoter sequences respond to environmental and growth factors and regulate expression of genes. In order to understand the possible response of PbPIN genes, the genomic sequences with $2 \mathrm{~kb}$ upstream of the translational start site (ATG) of the PbPIN genes were obtained from NCBI and analyzed in the Plant CARE database. More than 80 cis-acting elements in the promoter of PbPINs were detected, of which, 33 from at least seven promoters were analyzed (Table 3). On the basis of their functions, the identified cis-regulatory elements were classified into five groups. These comprised the core promoter elements, such as CAAT-box and TATA-box, existing in all promoters of PbPINs, hormone responsive related elements, such as TCA (salicylic acid related), CGTCA-motif, TGACG-motif (MeJA related), ABRE (abscisic acid related), GARE-motif, P-box, TATC-box (gibberellin related), and ERE (ethylene related), detected in at least eight promoters, stress-related elements (HSE, MBS, TC-rich repeats, and ARE), contained in more than seven promoters, light responsive related elements, such as Box4, G-box, GT1-motif, GAG-motif, Sp1, Box I, and AAGAA-motif, existing in at least seven promoters, and development related elements, such as circadian, Skn-1_motif, and O2-site, detected in almost everyone. The putative functions of cis-elements are listed in Table 3. These results indicated that the role of PbPINs might be closely related to plant growth and development, as well as response to endogenesis and exogenesis signals. 
Table 3. Cis-elements present in more than seven promoters of PIN genes in pear.

\begin{tabular}{|c|c|c|c|}
\hline Cis-Elements & Number of Gene & Functions of the Cis-Elements & Cis-Elements Types \\
\hline AE-box & 8 & part of a module for light response & Light responsive \\
\hline Box 4 & 12 & part of a conserved DNA module involved in light responsiveness & Light responsive \\
\hline G-Box & 11 & cis-acting regulatory element involved in light responsiveness & Light responsive \\
\hline G-box & 10 & cis-acting regulatory element involved in light responsiveness & Light responsive \\
\hline GA-motif & 7 & part of a light responsive element & Light responsive \\
\hline GT1-motif & 11 & light responsive element & Light responsive \\
\hline TCT-motif & 8 & part of a light responsive element & Light responsive \\
\hline ATCT-motif & 9 & part of a conserved DNA module involved in light responsiveness & Light responsive \\
\hline GAG-motif & 13 & part of a light responsive element & Light responsive \\
\hline Sp1 & 11 & light responsive element & Light responsive \\
\hline Box I & 12 & light responsive element & Light responsive \\
\hline CATT-motif & 8 & part of a light responsive element & Light responsive \\
\hline I-box & 7 & part of a light responsive element & Light responsive \\
\hline AAGAA-motif & 11 & & Light responsive \\
\hline ABRE & 7 & cis-acting element involved in the abscisic acid responsiveness & Hormone responsive \\
\hline CGTCA-motif & 11 & cis-acting regulatory element involved in the MeJA-responsiveness & Hormone responsive \\
\hline TGACG-motif & 11 & cis-acting regulatory element involved in the MeJA-responsiveness & Hormone responsive \\
\hline TCA-element & 11 & cis-acting element involved in salicylic acid responsiveness & Hormone responsive \\
\hline GARE-motif & 7 & gibberellin-responsive element & Hormone responsive \\
\hline P-box & 7 & gibberellin-responsive element & Hormone responsive \\
\hline ERE & 7 & ethylene-responsive element & Hormone responsive \\
\hline HSE & 11 & cis-acting element involved in heat stress responsiveness & Stress responsive \\
\hline MBS & 9 & MYB binding site involved in drought-inducibility & Stress responsive \\
\hline TC-rich repeats & 14 & cis-acting element involved in defense and stress responsiveness & Stress responsive \\
\hline ARE & 8 & cis-acting regulatory element essential for the anaerobic induction & Stress responsive \\
\hline Box-W1 & 8 & fungal elicitor responsive element & Stress responsive \\
\hline W box & 8 & wounding and pathogen response & Stress responsive \\
\hline circadian & 13 & cis-acting regulatory element involved in circadian control & development related elements \\
\hline Skn-1_motif & 13 & cis-acting regulatory element required for endosperm expression & development related elements \\
\hline O2-site & 7 & cis-acting regulatory element involved in zein metabolism regulation & development related elements \\
\hline CAAT-box & 14 & common cis-acting element in promoter and enhancer regions & promoter related elements \\
\hline TATA-box & 14 & core promoter element around -30 of transcription start & promoter related elements \\
\hline Unnamed__1 & 8 & $60 \mathrm{~K}$ protein binding site & site-binding related elements \\
\hline
\end{tabular}

Note: More than 80 cis-elements in the promoter of 14 PbPINs were detected. We displayed 33 cis-elements, which were detected in at least seven promoters. 


\subsection{Expression of PbPINs in Different Organs}

In order to determine the relationship of PINs expression and dwarfing ability of Pyrus rootstocks, we detected the expression of PbPINs in different organs, including shoot tips, stems, leaves, flowers, and fruits, in vigorous rootstock 'QN101' and dwarfing rootstock 'OHF51', through qRT-PCR technique. As shown in Figure 5, there was no significantly difference in the expression levels of most PbPINs in leaves and fruits between 'QN101' and 'OHF51.' Only PbPIN3-1/PbPIN4 and PbPIN1-4/PbPIN2-1 displayed higher expression levels in the two tissues. Differences in expression of PbPINs between 'QN101' and 'OHF51' were detected mainly in shoot tips, stems, and flowers. Based on the expression analysis and hierarchical clustering, PbPINs were divided into four major groups: Group 1 contained two genes (PbPIN1-4 and PbPIN2-1) with relatively higher expression levels in the fruits of 'OHF51' and 'QN101' and in the flowers of 'OHF51.' Group 2 contained four genes (PbPIN1-1, PbPIN8, PbPIN1-2, and PbPIN5-2) whose expression levels were obviously higher in the stem of 'QN101' than 'OHF51,' and minor differences were detected in leaves and shoot tips between 'QN101' and 'OHF51.' Group 3 contained four genes (PbPIN1-3, PbPIN2-2, PbPIN5-1, and PbPIN6) with relatively higher expression levels in the shoot tips of 'OHF51.' The expression of PIN2-2 in stems, and PIN1-3 in flowers was higher in 'OHF51' than 'QN101.' Group 4 contained four genes (PbPIN4, PbPIN3-1, PbPIN3-2, and PbPIN3-3), and relatively higher expression levels were detected in the shoot tip of 'OHF51' and the flowers of 'QN101.' Higher expression levels of PbPIN3-1 and PbPIN4 were also detected in leaves of 'QN101' and 'OHF51.' These results showed the organ-dependent differences in the expression of PbPINs. In the tissues where auxin is transported (shoot tip and stem), the expression levels of PbPINs were different in 'QN101' and 'OHF51,' suggesting that there might be some relationship between dwarfing ability and auxin polar transport in pear stocks.

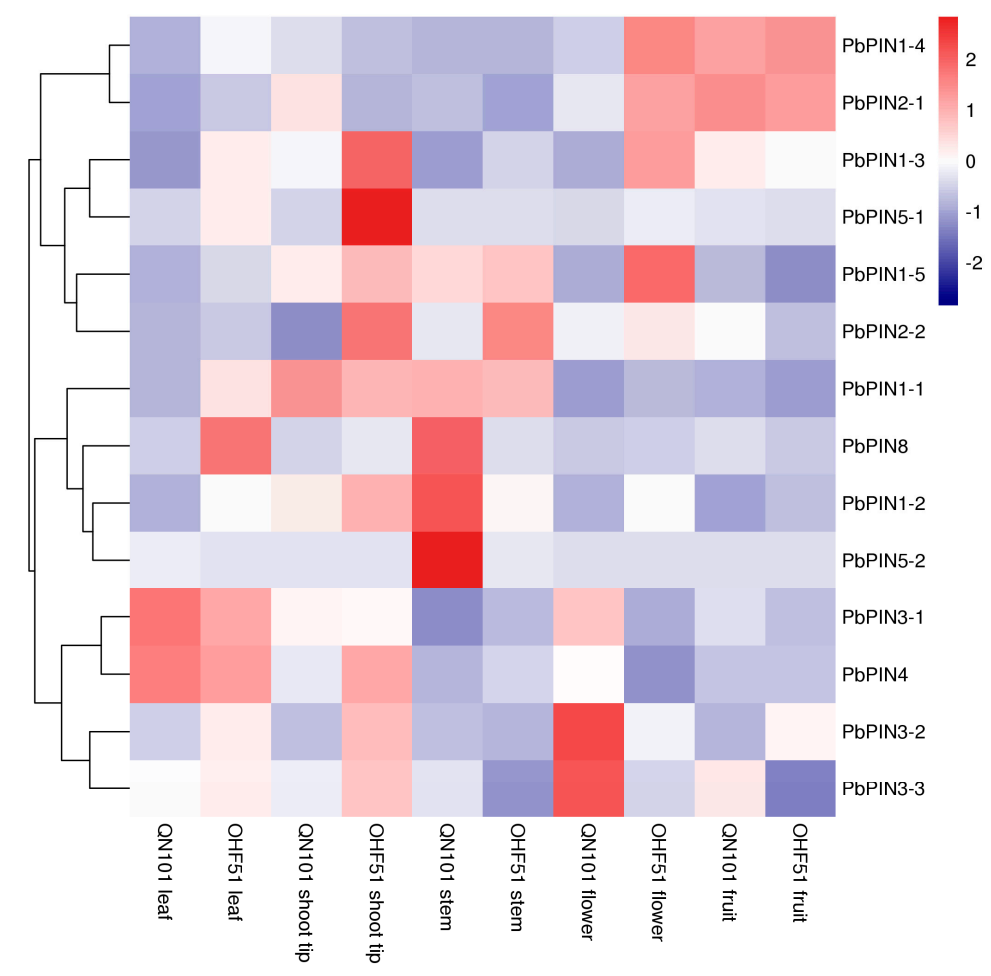

Figure 5. Heat map of the expression level of $14 \mathrm{PbPINs}$ in different organs. The tissues used for the expression profiling are indicated at the bottom of each column. A cluster dendrogram is shown on the left. PINs are divided into four major groups based on their expression. The color key at the top right corner represents the Z-score values transformed from log2-based expression values obtained by qRT-PCR. 


\section{Discussion}

In commercial apple production, dwarfing rootstocks have been widely used as they can successfully reduce scion vigor, necessitating high-density planting. In pear production, quince (Cydonia oblonga) is successfully used as the dwarfing rootstock of Pyrus communis L. Furthermore, a series of other Pyrus dwarfing stocks have also been reported, such as Old Home $\times$ Farmingdale clonal rootstocks [50]. However, the underlying mechanism of rootstock-induced dwarfing remains unclear.

In apple, it has been reported that the contents, transport, and signaling of hormones may play roles in rootstock/interstock-induced dwarfing [9-13]. In leaves and branch-barks of scions grafted on the dwarfing interstocks, IAA content was lower than that of vigorous combinations. However, when the interstock bark was substituted by invigorating interstock, the IAA content gradually restored to a standard tree's level $[15,16]$. When auxin transport in vigorous stocks was inhibited by polar auxin inhibitor, zeatin level in root and shoot growth declined dramatically [17]. The results helped to analyze the dwarfing mechanism of rootstocks or interstocks in fruit trees. Thus, at present, the best-supported dwarfing model proposes that lower expression of the PIN genes in stem bark tissue leads to the decrease of auxin transport, thereby reduces root growth and synthesis of cytokinin supplied to the scion, and in turn limited the growth of the scion $[17,37,51]$. However, research on the relationship of PIN genes and dwarfing ability in fruit trees are scarce, especially in Pyrus plants.

In the present study, we first collected the fruit quality and tree structural data from two different grafting combinations, 'Xueqing'/'QN101'/'Douli' and 'Xueqing'//OHF51'/'Douli,' at 2 years after grafting, which verified the dwarfing ability on the 'Xueqing' variety of stock 'OHF51' (Table 1). Considering the functions of PIN genes in dwarfing ability of apple rootstock and interstock, as well as the importance of pear production, we performed genome-wide comprehensive analysis of the pear PIN gene family, based on the white pear genomic data (NCBI; http://www.ncbi.nlm.nih.gov/).

Fourteen members of the PIN family have been found (Figure 1). In Arabidopsis [26], Oryza sativa [34], and Populus trichocarpa [46], as well as almost more than 30 plant species, PIN genes have been identified by genome-wide approaches. The lowest number of PIN genes is four, which is reported in Marchantia polymorpha, in contrast to the highest reported number of PIN genes (23) reported in Glycine max [43]. It has been suggested that the expanding PIN gene family may result from genome duplication. The number of PIN genes in pear is around twice of that in Arabidopsis. Depending on the phylogenetic analysis, Arabidopsis homologous genes widely exist in the pear genome, suggesting that some PIN proteins in Arabidopsis and pear may originate from one or more common genes. At the same time, PbPIN1s share low homology with AtPINs in Arabidopsis; perhaps merely because of the evolution of individual PIN families in Arabidopsis and pear. These phenomena are also found in other species $[15,42,44]$. PINs were categorized into four major subgroups depending on the neighbor-joining algorithm. Phylogenetic tree analysis with 99 PIN proteins from eight plant species showed that PINs could be divided into seven groups [43]. In Citrullus lanatus, phylogenetic tree analysis of 11 CIPIN proteins and eight AtPIN proteins grouped these proteins into five subfamilies [15]. The specificity of these groups in different species may result from the plant evolution.

The exon-intron diversification is also considered to play a role in the evolution of some gene family. Through exon-intron analysis, we found that seven out of 14 PbPINs contained six exons, and five of them belonged to subgroup I. This conserved exon-intron organization of PIN genes was also found in other plant species $[15,31,34,41,46]$. Meanwhile, PbPIN4 in subgroup I contained seven exons; PbPIN1-3/PbPIN1-4 in subgroup II and PbPIN5-1/PbPIN5-2 in subgroup IV contained eight exons and five and three exons, respectively. PbPIN6 belonging to subgroup III contained eight exons. Although differences existed, members in the same phylogenetic clade, especially the paralogous genes, possessed similar exon-intron structures, such as PbPIN2-1/PbPIN2-2 and PbPIN3-2/PbPIN3-3, as well as PbPIN5-1/PbPIN5-2. Therefore, it was speculated that the differences were not an occasional mutation event, but an evolution pattern of plant PIN genes. However, PbPIN4 and PbPIN3-1 in subgroup I exhibited obviously different exon-intron structure. In addition, we also found two specific members: PbPIN5-1, which had a long untranslated region, PbPIN6, containing a relatively longer 
intron. Thus, diversity might still exist in highly conserved genes because of the evolution. At the same time, we compared the exon/intron number between Arabidopsis and pear. In Arabidopsis, most of the AtPINs have six exons and five introns, except for AtPIN2 and AtPIN5, with nine and four exons. Among the orthologous genes between Arabidopsis and pear, such as PIN5s, PIN8s, PIN2s, and PIN6s, the exon/intron numbers are inconsistent. Additionally, among the paralogous genes in pear, PbPIN3-2/PbPIN3-3, PbPIN2-1/PbPIN2-2, and PbPIN5-1/PbPIN5-2, the exon/intron numbers are consistent. It has been regarded that the existence of introns facilitated gene evolution by exon shuffling, allowed the production of multiple proteins from a single gene through alternative splicing, as well as playing a role in gene regulation [52,53]. Thus, we speculate that during the evolution of pear, a series of intron deletion and insertion, gene duplication happened in PIN genes, which might cause changes in gene expression and protein function.

In Arabidopsis and other plant species, PIN proteins have two highly conserved hydrophobic segments located at $\mathrm{N}$ - and $\mathrm{C}$ - termini that are linked by a central hydrophilic loop with heterogeneity. Depending on the length divergence of central hydrophilic loop, PIN proteins are grouped into long and short PINs. In Arabidopsis, AtPIN1, 2, 3, 4, and 7, which have a long hydrophilic loop between two transmembrane regions, form the canonical PIN proteins, defined as long PIN proteins [29], and are mostly localized to the plasma membrane. The non-canonical PIN proteins (AtPIN5, 6 and 8) have a shorter central hydrophilic loop. They are known as short PIN proteins. AtPIN5 and AtPIN8 are localized to the endoplasmic reticulum (ER), while AtPIN6 has been reported to be localized at the ER and the plasma membrane [30]. In our research, the PbPINs all have two highly conserved hydrophobic segments located at N- and C-terminus, which are linked by a central hydrophilic loop. Similar to other plant PINs, the pear PINs can also be grouped into long PINs, which comprise 11 members (578-666 amino acids in length), and short PINs, which comprise PIN5-1, PIN5-2, and PIN8 (353-377 amino acids in length) according to the predicted protein length (Table 2). In addition, the length of the central hydrophilic loop is around 300 amino acids for members of the long PINs, and 50-100 amino acids for members of the short PINs. In Arabidopsis, the long PIN proteins localized in plasma membrane are responsible for the cell-to-cell auxin polar transport [23-25], while the majority of the ER-localized short ones are involved in intracellular regulation of auxin homeostasis $[24,25,29,48]$. Therefore, it can be speculated that the protein lengths are related to the location and function, which may help us to predict the function of orthologous genes of PINs in pear.

Several studies have shown that PIN genes are involved in plant growth and development processes. In the present study, the transcript levels of 14 PbPINs were analyzed in five different tissues of 'QN101' and 'OHF51,' the vigorous and dwarfing rootstocks. Our results showed that PbPINs had a variety of expression patterns in different organs of different interstocks, mainly in shoot tips, stems, and flowers of 'OHF51' or 'QN101.' Similar expression patterns have been detected in other species. In rice, OsPIN5 $a$ and OsPIN5c are highly expressed in leaves, shoot apex, and panicle, while OsPIN5b is mainly expressed in young panicles [34]. ZmPIN1b is highly expressed during female inflorescence development in maize [35]. In watermelon, the CIPINs are mainly expressed in mature leaves and stems, and weakly expressed in flowers [15]. More than half of the NtPIN genes are highly expressed in stems, NtPIN5a and NtPIN5b were highly expressed in flowers [44]. The variety of expression patterns in different organs of different interstocks suggested that the PIN gene family in pear stock might be related to different functions in growth and development process.

Expression patterns analysis of the 14 PbPINs showed that some genes belonging to the same subgroup depending on the neighbor-joining algorithm, such as PbPIN3-1, PbPIN3-2, PbPIN3-3, and PbPIN4 in subgroup I, had similar expression patterns, suggesting that these genes probably obtained redundant functions during evolution. However, some genes that belong to the same subgroup had divergent expression patterns, such as the paralogous genes, PbPIN2-1 and PbPIN2-2, indicating that these genes obtained unique functions during evolution. In addition, some similar expression of PbPINs from different subgroup suggested the functional complementation of PbPINs, such as PbPIN1-4 and PbPIN2-1, PbPIN1-2, and PbPIN5-2. 
Previous studies found that dwarfing is due to the lower expression of the MdPIN1b gene in stem bark tissue of dwarfing apple interstock, blocking the downward transport of auxin [17]. In Pyrus communis, the expression of a PcPIN-L is significantly lower in the dwarf-type pears than in standard-type pears. Overexpression of the PCPIN-L in tobacco enhances the growth of the stems and the roots [54]. In contrast, overexpression of OsPIN2 dwarfs the plant height [37]. ZmPIN1a overexpression also reduces plant height and internode length [51]. Between 'QN101' and 'OHF51,' some PbPINs displayed different expression patterns. The expression level of PbPIN8 was higher in leaves of 'OHF51' than of 'QN101.' In flowers, the expression of PbPIN3-1, PbPIN3-2, and PbPIN3-3 was lower in 'OHF51,' in contrast to the expression level of PbPIN1-3, PbPIN1-4, and PbPIN2-1. In addition, a series of genes, such as PbPIN1-2 in group 2, group 3's PbPIN1-3/PbPIN2-2/PbPIN5-1/PbPIN6, and PbPIN4/PbPIN3-2/ PbPIN3-3 in group 4, showed obviously higher expression levels in shoot tips of 'OHF51' than that of 'QN101.' The expression of PbPIN8, PbPIN1-2, and PbPIN5-2 in stem of 'OHF51' was also lower than that of 'QN101.' It should be noted that PbPIN2-2 showed a higher expression level in both the shoot tips and stem of 'OHF51' than that of 'QN101.' We believe that shoot tips and stems are the tissues where auxin is transported. The differences in expression in shoot tips and stems between 'QN101' and 'OHF51' suggest that the ability of auxin polar transport differs between vigorous and dwarfing stocks, and there may be some relationship between dwarfing ability and auxin polar transport in pear stocks.

\section{Materials and Methods}

\subsection{Plant Materials and Treatments}

The vigorous and dwarfing interstocks, 'QN101' (Pyrus betulifolia) and 'OHF51' (Pyrus communis), were grafted on 'Douli' (Pyrus calleryana) seedlings, respectively, which were cultivated in the pear experimental plot of Qingdao Agricultural University. In the following spring, cultivar 'Xueqing' (Pyrus bretschneideri $\times$ Pyrus pyrifolia) was grafted onto the interstocks. Data of tree growth and fruit quality were collected 2 years later. Tree height was recorded after the stop of the annual growth and before pruning. Interstock diameter was evaluated at the middle site; and scion and rootstock diameters were recorded at $5 \mathrm{~cm}$ above and below the interstock, respectively. The TSS of the fruit was determined utilizing an ATAGO saccharimeter that had been standardized with distilled water.

'QN101' and 'OHF51' grafted on 'Douli' were used for the expression profiling analysis. Three samples of shoot tips, leaves, flowers, stems, and roots were collected from pear trees grown in the orchard of Qingdao Agriculture University on 15 April 2017, whereas three samples of fruits were collected on 15 October 2017. All samples were immediately frozen in liquid nitrogen and stored at $-80{ }^{\circ} \mathrm{C}$ until RNA extraction.

\subsection{Identification and Characteristics of PINs}

In order to recognize the auxin efflux carriers (PINs) in Chinese white pear, we searched the corresponding Pear Genome Project (http://peargenome.njau.edu.cn/) [55] and the National Center for Biotechnology Information (NCBI; http://www.ncbi.nlm.nih.gov/) by BLAST (Basic Local Alignment Search Tool) search tools BLASTP and BLASTN [56], using AtPINs sequences as queries which were identified from the TAIR (The Arabidopsis Information Resource) database v10.0 (http: //www.arabidopsis.org/). Multiple alignment of the full-length deduced amino acid sequences of PIN proteins was then performed with Clustal X [57], and redundant sequences and different transcripts of the same gene were discarded. SMART (http://smart.embl-heidelberg.de/) [58] and Pfam (http://pfam.san ger.ac.uk/) [59] were used to predict the domain composition of PbPINs. Protein molecular weights $(\mathrm{Da})$ and isoelectric points $(\mathrm{pI})$ were analyzed using the ExPASy proteo- mics server [60]. Alignment of coding sequences with the corresponding genomic DNA sequences was used to determine the CDS-intron structure of PbPINs, and the graphs of exon-intron structures were generated using the Gene Structure Display Server (http://gsds.cbi.pku.edu.cn/) [61]. 


\subsection{Sequence Alignment and Phylogenetic Analysis}

Multiple sequence alignments of PIN sequences from Chinese white pear (Pyrus bretschneideri) and Arabidopsis, which were created using ClustalX in MEGA 7.0 with the default parameters [62] were used as the input for the neighbor-joining algorithm in MEGA7.0 to construct phylogenetic trees. Bootstrap analysis was conducted with 1000 replicates to assess the statistical support for each node. Based on the phylogenetic trees, we designated PbPINs following the nomenclature system of the PIN family from Arabidopsis.

\subsection{Sequence Conservation Analysis}

Conserved sequences were obtained by SMART and Pfam using the multiple sequence alignment of PbPINs that were created using Clustal X2. The protein trans-membrane topology was predicted using the TMHMM Server v2.0 (http://www.cbs.dtu.dk/services/TMHMM/).

\subsection{Cis-Acting Regulatory Element Prediction in Promoters Regions of PbPINs}

To investigate the putative cis-acting elements in the promoter sequences of PbPINs, $2000 \mathrm{bp}$ genomic DNA sequences upstream of the translational start site (ATG) were obtained from the Chinese white pear genome. The upstream sequences were subsequently analyzed and annotated using PlantCARE (http://bioinformatics.psb.ugent.be/webtools/plantcare/html/) [63] for the presence of various cis-acting elements [64].

\subsection{RNA Extraction and Quantitative Real-Time PCR ( $q R T-P C R)$ Analysis}

Total RNA was extracted using the RNAprep Pure Plant kit (Tiangen, Beijing, China), following the manufacturer's instructions. cDNAs were synthesized from moderate total RNA using a PrimeScriptTM RT reagent kit with gDNA Eraser (TaKaRa, Tokyo, Japan). qRT-PCR was performed using the Roche 480 real-time PCR system at the standard mode with the FastStart Essential DNA Green Master kit. All reactions were performed in triplicate at a volume of $20 \mu \mathrm{L}$, containing $1 \mu \mathrm{L}$ of diluted cDNA, and the pear actin gene was used as an internal control. The qRT- PCR amplification was carried out as follows: $95^{\circ} \mathrm{C}$ for $5 \mathrm{~min}, 45$ cycles at $95^{\circ} \mathrm{C}$ for $15 \mathrm{~s}, 60^{\circ} \mathrm{C}$ for $30 \mathrm{~s}$, and $72{ }^{\circ} \mathrm{C}$ for $30 \mathrm{~s}$. PbPIN gene-specific primers for quantitative PCR are listed in the Table 4. The relative expression levels of PbPINs were calculated using the $2^{-\Delta \Delta C T}$ method. All qPCR analyses have three biological replicates and two technical replicates.

Table 4. The primer of the RT-PCR reaction.

\begin{tabular}{|c|c|c|c|}
\hline Name & Sequence & Name & Sequence \\
\hline \multirow[t]{2}{*}{ Actin } & U: CCCAGAAGTGCTCTTCCAAC & PbPIN3-2 & U: АТTTТАССССТССССТСТТСТ \\
\hline & D: TTGATCTTCATGCTGCTTGG & & D: AATATCATCGCCACGTACAGC \\
\hline \multirow{2}{*}{ PbPIN1-1 } & U: ATTGTTCCCTTTGTCTTTGC & PbPIN3-3 & U: GCCATTCTTAGCACTGCGGTTAT \\
\hline & D: TTCTCATCATGTTTGCGTTT & & D: GGCGGATTCTTCGTTGATCTTCAT \\
\hline \multirow{2}{*}{ PbPIN1-2 } & U: CCCAGAAACAGAGCAATAAGA & PbPIN4 & U: CATTCTTAGCACTGCGGTTATT \\
\hline & D: TGGAGATGAAGTGGAAGGAGA & & D: TCGTTCTCCATCGTACTTAATCTA \\
\hline \multirow[t]{2}{*}{ PbPIN1-3 } & U: GATTGTTCCGTTTGTCTT & PbPIN5-1 & U: TCTGGGTAGTCCTCAAGTCC \\
\hline & D: ATATTTCCTCCACGTCTC & & D: GTCAGCCGCTATGAACCT \\
\hline \multirow[t]{2}{*}{ PbPIN1-4 } & U: TTGATTTTCCTTCTGATTTCGG & PbPIN5-2 & U: ATTAATTACCAGTTGTGCTCCAA \\
\hline & D: GAAGATTTTCCACCATTTCACG & & D: ATCAACATGTGCAGTGAATTCGA \\
\hline \multirow[t]{2}{*}{ PbPIN2-1 } & U: GCCTTGCCTGTAACAATACT & PbPIN6 & U: GTTTCCTTACCAGTGACGCTCCT \\
\hline & D: ATCAAGGTACTCAGTTGTCACAT & & D: CATGTACTAATATGACACCAGACCC \\
\hline \multirow[t]{2}{*}{ PbPIN2-2 } & U: ACGCGGACATACTTAGCACTG & PbPIN8 & U: CATCCAGACATTTTGAGCAC \\
\hline & D: TGAACACTGAATCCAATGGCA & & D: GTAAACATATCAGCCATATTACA \\
\hline \multirow[t]{2}{*}{ PbPIN3-1 } & U: CTATTGTCCAGGCTGCTCTTC & & \\
\hline & D: TTCTTCGTTTTCCTAGCTTTGT & & \\
\hline
\end{tabular}


Author Contributions: Data curation, L.Q., L.C., C.W., and Y.Y.; Funding acquisition, R.W.; Software, S.Z., J.L., D.L., and J.S.; Writing-review and editing, R.W. All authors have read and agree to the published version of the manuscript.

Funding: This work was supported by the China Agricultural Research System (CARS-29-07), the National Natural Science Foundation of China (31572109), and the Special Foundation for Distinguished Taishan Scholar of Shangdong Province.

Conflicts of Interest: The authors declare no conflict of interest.

\section{References}

1. Davis, T.D.; Curry, E.A.; Steffens, G.L. Chemical regulation of vegetative growth. Crit. Rev. Plant Sci. 1991, 10, 151-188. [CrossRef]

2. Basak, A. Growth regulation of pome and stone fruit trees by use of prohexadione-Ca. Acta Hortic. 2000, 514, 41-50. [CrossRef]

3. Bubán, T. The use of benzyladenine in orchard fruit growing: A mini review. Plant Growth Regul. 2000, 32, 381-390. [CrossRef]

4. Medjdoub, R.; Val, J.; Blanco, A. Prohexadione-Ca inhibits vegetative growth of 'Smoothee Golden Delicious' apple trees. Sci. Hortic. 2004, 101, 243-253. [CrossRef]

5. Reighard, G.L.; Ouellette, D.; Rauh, B.; Bridges, W., Jr. New rootstock cultivars for peach Influence growth, yield and fruit quality. Acta Hortic. 2014, 1058, 517-522. [CrossRef]

6. Webster, A.D. A review of fruit tree rootstock research and development. Acta Hortic. 1997, 451, 53-73. [CrossRef]

7. Webster, T. Dwarfing rootstocks:past, present and future. Compact Fruit Tree 2002, 35, 67-72.

8. Atkinson, C.J.; Else, M.A.; Taylor, L. Root and stem hydraulic conductivity as determinants of growth potential in grafted trees of apple (Malus pumila Mill.). J. Exp. Bot. 2003, 54, 1221-1229. [CrossRef]

9. Lockard, R.G.; Schneideri, G.W. Stock and scion growth relationships and the dwarfing mechanism in apple. Hort. Rev. 1981, 3, 315-375.

10. Tustin, S.; Warrington, I.; Woolley, D.; van Hooijdonk, B.M. Rootstocks modify scion architecture, endogenous hormones, and root growth of newly grafted 'royal gala' apple trees. J. Am. Soc. Hortic. Sci. 2011, 136, 93-102.

11. Richards, D.; Thompson, W.K.; Pharis, R.P. The influence of dwarfing interstocks on the distribution and metabolism of xylem-applied [3H]Gibberellin A4 in apple. Plant Physiol. 1986, 82, 1090-1095. [CrossRef] [PubMed]

12. Van Hooijdonk, B.M.; Woolley, D.J.; Warrington, I.J.; Tustin, D.S. Initial alteration of scion architecture by dwarfing apple rootstocks may involve shoot-root-shoot signalling by auxin, gibberellin, and cytokinin. J. Hortic. Sci. Biotechnol. 2010, 85, 59-65. [CrossRef]

13. Kamboj, J.S.; Browning, G.; Blake, P.S.; Quinlan, J.D.; Baker, D.A. GC-MS-SIM analysis of abscisic acid and indole-3-acetic acid in shoot bark of apple rootstocks. Plant Growth Regul. 1999, 28, 21-27. [CrossRef]

14. Tworkoski, T.; Fazio, G. Effects of size-controlling apple rootstocks on growth, abscisic acid, and hydraulic conductivity of scion of different vigor. Int. J. Fruit Sci. 2015, 15, 369-381. [CrossRef]

15. Yu, C.; Dong, W.; Zhan, Y.; Huang, Z.A.; Li, Z.; Kim, I.S. Genome-wide identification and expression analysis of ClLAX, ClPIN and ClABCB genes families in Citrullus lanatus under various abiotic stresses and grafting. BMC Genet. 2017, 18, 33. [CrossRef]

16. Li, K.; Kamiya, T.; Fujiwara, T. Differential roles of PIN1 and PIN2 in root meristem maintenance under low-B conditions in Arabidopsis thaliana. Plant Cell Physiol. 2015, 56, 1205-1214. [CrossRef]

17. Zhang, H.; An, H.S.; Wang, Y.; Zhang, X.Z.; Han, Z.H. Low expression of PIN gene family members is involved in triggering the dwarfing effect in M9 interstem but not in M9 rootstock apple trees. Acta Physiol. Plant. 2015, 37, 104. [CrossRef]

18. Swarup, R.; Kargul, J.; Marchant, A.; Zadik, D.; Rahman, A.; Mills, R.; Yemm, A.; May, S.; Williams, L.; Millner, P.; et al. Structure-function analysis of the presumptive Arabidopsis auxin permease AUX1. Plant Cell 2004, 16, 3069-3083. [CrossRef] 
19. Petrasek, J.; Mravec, J.; Bouchard, R.; Blakeslee, J.J.; Abas, M.; Seifertova, D.; Wisniewska, J.; Tadele, Z.; Kubes, M.; Covanova, M.; et al. PIN proteins perform a rate-limiting function in cellular auxin efflux. Science 2006, 312, 914-918. [CrossRef]

20. Cho, M.; Lee, S.H.; Cho, H.T. P-glycoprotein4 displays auxin efflux transporter-like action in Arabidopsis root hair cells and tobacco cells. Plant Cell 2007, 19, 3930-3943. [CrossRef]

21. Li, H.L.; Zhang, H.; Yu, C. Possible roles of auxin and zeatin for initiating the dwarfing effect of M9 used as apple rootstock or interstock. Acta Physiol. Plant 2012, 34, 235-244. [CrossRef]

22. Friml, J. Efux-dependent auxin gradients establish the apical-basal axis of Arabidopsis. Nature 2003, 426, 147-153. [CrossRef] [PubMed]

23. Tanaka, H.; Dhonukshe, P.; Brewer, P.B.; Friml, J. Spatiotemporal asymmetric auxin distribution: A means to coordinate plant development. Cell. Mol. Life Sci. 2006, 63, 2738-2754. [CrossRef] [PubMed]

24. Friml, J. Subcellular trafficking of PIN auxin efflux carriers in auxin transport. Eur. J. Cell Biol. 2010, 89, 231-235. [CrossRef]

25. Viaene, T.; Delwiche, C.F.; Rensing, S.A.; Friml, J. Origin and evolution of PIN auxin transporters in the green lineage. Trends Plant Sci. 2013, 18, 5-10. [CrossRef]

26. Paponov, I.A.; Teale, W.D.; Trebar, M.; Blilou, I.; Palme, K. The PIN auxin efflux facilitators: Evolutionary and functional perspectives. Trends Plant Sci. 2005, 10, 170-177. [CrossRef]

27. Ding, Z.; Wang, B.; Moreno, I.; Duplakova, N.; Simon, S.; Carraro, N.; Reemmer, J.; Pencik, A.; Chen, X.; Tejos, R.; et al. ER-localized auxin transporter PIN8 egulates auxin homeostasis and male gametophyte development in Arabidopsis. Nat. Commun. 2012, 3, 941. [CrossRef]

28. Mravec, J.; Skupa, P.; Bailly, A.; Hoyerova, K.; Krecek, P.; Bielach, A.; Petrasek, J.; Zhang, J.; Gaykova, V.; Stierhof, Y.D. Subcellular homeostasis of phytohormone auxin is mediated by the ER localized PIN5 transporter. Nature 2009, 459, 1136-1140. [CrossRef]

29. Bennett, T.; Brockington, S.F.; Rothfels, C.; Graham, S.W.; Stevenson, D.; Kutchan, T.; Rolf, M.; Thomas, P.; Wong, G.K.; Leyser, O.; et al. Paralogous radiations of PIN proteins with multiple origins of noncanonical PIN structure. Mol. Biol. Evol. 2014, 31, 2042-2060. [CrossRef]

30. Simon, S.; Skupa, P.; Viaene, T.; Zwiewka, M.; Tejos, R.; Klima, P.; Carna, M.; Rolcik, J.; De Rycke, R.; Moreno, I.; et al. PIN6 auxin transporter at endoplasmic reticulum and plasma membrane mediates auxin homeostasis and organogenesis in Arabidopsis. New Phytol. 2016, 211, 65-74. [CrossRef]

31. Krecek, P.; Skupa, P.; Libus, J.; Naramoto, S.; Tejos, R.; Friml, J.; Zazimalova, E. The PIN-FORMED (PIN) protein family of auxin transporters. Genome Biol. 2009, 10, 249. [CrossRef] [PubMed]

32. Robert, H.S.; Grones, P.; Stepanova, A.N.; Robles, L.M.; Lokerse, A.S.; Alonso, J.M.; Weijers, D.; Friml, J. Local auxin sources orient the apical-basal axis in Arabidopsis embryos. Curr. Biol. 2013, 23, 2506-2512. [CrossRef] [PubMed]

33. Ganguly, A.; Lee, S.H.; Cho, M.; Lee, O.R.; Yoo, H.; Cho, H.T. Differential auxin-transporting activities of PIN-FORMED proteins in Arabidopsis root hair cells. Plant Physiol. 2010, 153, 1046-1061. [CrossRef] [PubMed]

34. Wang, J.R.; Hu, H.; Wang, G.H.; Li, J.; Chen, J.Y.; Wu, P. Expression of PIN genes in rice (Oryza sativa L.): Tissue specificity and regulation by hormones. Mol. Plant. 2009, 2, 823-831. [CrossRef] [PubMed]

35. Yue, R.; Tie, S.; Sun, T.; Zhang, L.; Yang, Y.; Qi, J. Genome-wide identification and expression profiling analysis of ZmPIN, ZmPILS, ZmLAX and ZmABCB auxin transporter gene families in maize (Zea mays L.) under various abiotic stresses. PLOS ONE 2015, 10, e0118751. [CrossRef]

36. Peng, H.; Peng, Z.; Wang, L.; Zhang, Y.; Wang, X.; Hui, X. The PIN gene family in cotton (gossypium hirsutum): Genome-wide identification and gene expression analyses during root development and abiotic stress responses. BMC Genom. 2017, 18, 507.

37. Chen, Y.; Fan, X.; Song, W.; Zhang, Y.; Xu, G. Over-expression of OsPIN2 leads to increased tiller numbers, angle and shorter plant height through suppression of OsLAZY1. Plant Biotechnol. J. 2012, 10, 139-149. [CrossRef]

38. Zhang, Q.; Li, J.; Zhang, W.; Yan, S.; Wang, R.; Zhao, J.; Li, Y.; Qi, Z.; Sun, Z.; Zhu, Z. The putative auxin efflux carrier OsPIN3t is involved in the drought stress response and drought tolerance. Plant J. 2012, 72, 805-816. [CrossRef] 
39. Carraro, N.; Peer, W.A. Immunolocalization of PIN and ABCB transporters in plants. Methods Mol. Biol. 2016, 1398, 55-67.

40. Forestan, C.; Meda, S.; Varotto, S. ZmPIN1-mediated auxin transport is related to cellular differentiation during maize embryogenesis and endosperm development. Plant Physiol. 2010, 152, 1373-1390. [CrossRef]

41. Shen, C.; Bai, Y.; Wang, S.; Zhang, S.; Wu, Y.; Chen, M.; Jiang, D.; Qi, Y. Expression profile of PIN, AUX/LAX and $P G P$ auxin transporter gene families in Sorghum bicolor under phytohormone and abiotic stress. FEBS J. 2010, 277, 2954-2969. [CrossRef] [PubMed]

42. Pattison, R.J.; Catalá, C. Evaluating auxin distribution in tomato (Solanum lycopersicum) through an analysis of the PIN and AUX/LAX gene families. Plant J. 2012, 70, 585-598. [CrossRef] [PubMed]

43. Wang, Y.; Chai, C.; Valliyodan, B.; Maupin, C.; Annen, B.; Nguyen, H.T. Genome-wide analysis and expression profiling of the PIN auxin transporter gene family in soybean (glycine max). BMC Genom. 2015, 16, 1-13. [CrossRef] [PubMed]

44. Xie, X.; Qin, G.; Si, P.; Luo, Z.; Gao, J.; Chen, X. Analysis of Nicotiana tabacum PIN genes identifies NtPIN4 as a key regulator of axillary bud growth. Physiol. Plant. 2017, 160, 222-239. [CrossRef]

45. Gao, L.W.; Lyu, S.W.; Tang, J.; Zhou, D.Y.; Bonnema, G.; Xiao, D.; Hou, X.L.; Zhang, C.W. Genome-wide analysis of auxin transport genes identifies the hormone responsive patterns associated with leafy head formation in Chinese cabbage. Sci. Rep. 2017, 7, 42229. [CrossRef]

46. Liu, B.; Zhang, J.; Wang, L. A survey of Populus PIN-FORMED family genes reveals their diversified expression patterns. J. Exp. Bot. 2014, 65, 2437-2448. [CrossRef]

47. Fett-Neto, A.; Almeida, M.D.; Ruedell, C. Expression of auxin carrier genes during adventitious rooting in Eucalyptus globulus. BMC Proc. 2011, 5, 64. [CrossRef]

48. Friml, J.; Wisniewska, J.; Benkova, E.; Mendgen, K.; Palme, K. Lateral relocation of auxin efflux regulator PIN3 mediates tropism in Arabidopsis. Nature 2002, 415, 806-809. [CrossRef]

49. Cho, M.; Cho, H.T. The function of ABCB transporters in auxin transport. Plant Signal. Behav. 2013, 8, e22990. [CrossRef]

50. Zhu, L.H. Adventitious shoot regeneration of two dwarfing pear rootstocks and the development of a transformation protocol. J. Hortic. Sci. Biotech. 2000, 75, 745-752. [CrossRef]

51. Li, Z.; Zhang, X.; Zhao, Y.; Li, Y.; Zhang, G.; Peng, Z.; Zhang, J. Enhancing auxin accumulation in maize root tips improves root growth and dwarfs plant height. Plant Biotechnol. J. 2018, 16, 86. [CrossRef] [PubMed]

52. Rose, A.B. The effect of intron location on intron-mediated enhancement of gene expression in Arabidopsis. Plant J. 2005, 40, 744-751. [CrossRef] [PubMed]

53. Rose, A.B. Requirements for intron-mediated enhancement of gene expression in Arabidopsis. RNA 2002, 8, 1444-1453. [CrossRef] [PubMed]

54. Zheng, X.; Zhang, H.; Xiao, Y.; Wang, C.; Tian, Y. Deletion in the promoter of PcPIN-L affects the polar auxin transport in dwarf pear (Pyrus communis L.). Sci. Rep. 2019, 9. [CrossRef]

55. Wu, J.; Wang, Z.; Shi, Z.; Zhang, S.; Ming, R.; Zhu, S.; Khan, M.A.; Tao, S.; Korban, S.S.; Wang, H.; et al. The genome of the pear (Pyrus bretschneideri Rehd.). Genome Res. 2013, 23, 396-408. [CrossRef]

56. Altschul, S.F.; Madden, T.L.; Schäffer, A.A.; Zhang, J.; Zhang, Z.; Miller, W.; Lipman, D.J. Gapped BLAST and PSI-BLAST: A new generation of protein database search programs. Nucleic Acids Res. 1997, 25, 3389-3402. [CrossRef]

57. Jeanmougin, F.; Thompson, J.D.; Gouy, M.; Higgins, D.G.; Gibson, T.J. Multiple sequence alignment with Clustal, X. Trends Biochem. Sci. 1998, 23, 403-405. [CrossRef]

58. Schultz, J.; Milpetz, F.; Bork, P.; Ponting, C.P. Smart, a simple modular architecture research tool: Identification of signaling domains. PNAS 1998, 95, 5857-5864. [CrossRef]

59. Bateman, A. The Pfam protein families database. Nucleic Acids Res. 2004, 32, 138-141. [CrossRef]

60. Gasteiger, E.; Gattiker, A.; Hoogland, C.; Ivanyi, I.; Appel, R.D.; Bairoch, A. ExPASy: The proteomics server for in-depth protein knowledge and analysis. Nucleic Acids Res. 2003, 31, 3784-3788. [CrossRef]

61. Guo, A.Y.; Zhu, Q.H.; Chen, X.; Luo, J.C. GSDS: A gene structure display server. Hereditas 2007, 29, $1023-1026$. [CrossRef] [PubMed]

62. Kumar, S.; Stecher, G.; Tamura, K. MEGA7: Molecular evolutionary genetics analysis version 7.0 for bigger datasets. Mol. Biol. Evol. 2016, 33, 1870-1874. [CrossRef] 
63. Lescot, M.; Déhais, P.; Thijs, G.; Marchal, K.; Moreau, Y.; Van de Peer, Y.; Rombauts, S. PlantCARE, a database of plant cis-acting regulatory elements and a portal to tools for in silico analysis of promoter sequences. Nucleic Acids Res. 2002, 30, 325-327. [CrossRef] [PubMed]

64. Kenichi, H.; Yoshihiro, U.; Masao, I.; Tomoko, K. Plant cis-acting regulatory DNA elements (PLACE) database. Nucleic Acids Res. 1999, 27, 297-300. 